\title{
Coverage Area of Path Planning Mobility Model for Screening Post-Disaster Area
}

\author{
Intan Nabina Azmi, Yusnani Mohd Yussoff, Murizah Kassim, and Nooritawati Md Tahir
}

\begin{abstract}
Recently, drone has been widely used for numerous application. One of them includes research on a drone for efficient services with minimal cost and risk. Due to the limitation of drone battery lifetime, an optimum flight pattern is needed to avoid power loss during flying while being able to effectively scan the required area. Hence, this paper presents an analysis of drone optimum flying pattern to achieve the highest coverage for limited time and energy consumption. The study focuses on site surveying application intended for post-disaster area. The analysis was conducted using OMNeT++ simulator version 5.2.1. The outcome from the analysis shows that the percentage of the coverage area for Zigzag and Square patterns increase by $13.03 \%$ and $17.67 \%$ at $20 \mathrm{~m}$ height compared to $10 \mathrm{~m}$ height using DJI Phantom 3 Standard. Initial results from the numerical analysis also show that the specification of the camera and the height of the flying drone has a huge impact to gain a wider coverage area. The findings from the study help to identify the optimum camera technical specification for the search and rescue mission considering that post-hazardous situation which has a great deal of limitations to human access.
\end{abstract}

Index Terms - coverage area, field of view, flying pattern, mobility model

\section{INTRODUCTION}

$\mathrm{D}$ RONE technology has been introduced since 1800 s and has been employed by US military since 2001 [1]. Then, the technology evolved along with other technologies. Drone utilization has been begun by the military unit, at that point it starts to draw in individuals as another leisure activity who has passion in photographic [2], [3] and become commercial items. However, the benefit of using a drone in industry operation shows a good improvement as a medium of data collection for forensics [4], marine [5], agriculture [5] and networks [6], [7].

This manuscript is submitted on $18^{\text {th }}$ November 2020 and accepted on $14^{\text {th }}$ April 2021. This work was supported by the Ministry of Education (MOE) Malaysia under grant 600-IRMI/TRGS 5/3 (001/2019)-1, and the Research Management Centre (RMC) of Universiti Teknologi MARA.

Intan Nabina Azmi, Yusnani Mohd Yussof, Murizah Kassim and Nooritawati Md Tahir are with are with the School of Electrical Engineering, College of Engineering, Universiti Teknologi MARA, 40450 Shah Alam, Selangor (e-mail: intannabinaazmi@gmail.com, yusna233@uitm.edu.my,murizah@uitm.edu.my,nooritawati@ieee.org)

1985-5389/C 2021 The Authors. Published by UiTM Press. This is an open access article under the CC BY-NC-ND license (http://creativecommons.org /licenses/by-nc-nd/4.0/).
Due to its compact size, Drone becomes one of the smart devices with the attachment of several components such as camera, obstacle prevention, GPS, infrared thermometer [8] and gas sensor [9]. The combination of the camera and the compact size of the drone makes it accessible to all conditions and places where human access is severe. Using a drone, view of the hardly accessible area such in disaster environment is available remotely. The accessibility to the remote area, however, very much depends on the field of view (FOV) angle of the camera.

Recently, camera technology has advanced drastically due to the high demand and competition among smartphone developers. The significance of the FOV angle is to give a decent and wide view caught particularly for landscape pictures and recordings. The FOV angle likewise may be influenced by the distance between the focal point and the object [10], [11].

On the other hand, the flight pattern or mobility model is another important area to be investigated, especially for surveillance purposes. Without a proper path for drone deployment, there is a possibility that the drones will fly at a comparative point for various occasions thus, some area will not be covered. A systematic approach to drones not only helps to avoid collisions between drones but also helps to improve communication [12]. There are five distinct classes of versatility models explicitly irregular based, time-sensitive, way based, bunch based and geography-based [13].

This research is part of research work in [14]. After a disaster event, the military and other organization will immediately plot the operation to monitor the area safety and find survivors as soon as possible. A drone is the best equipment to survey the hazardous area but if the drone fly to 0 high the changes of human recognition is thin.

Therefore, in this paper, a mobility model with high coverage area is presented. We apply the flying pattern as studied in [15] with different camera models and heights. The chosen cameras are made dependent on their FOV angle specification and the availability of the drone itself. Next, a correlation between both camera will be made followed by the miscellaneous height of flight. Hence, along with a decent camera detail and appropriate height, the drone is able to scan a region at the greatest inclusion input. 


\section{RELATED WORK}

This section presents recent reviews on drone-related research that is relevant to communication model services, mobility model and the density of the drones. It is intended to identify current research in this area and the loopholes that exist.

\section{A. Communication Protocol}

In 1997, wireless standard IEEE 802.11 has been released with $2.4 \mathrm{GHz}$ oscillation rate, speed of $2 \mathrm{Mbps}$ and transmission range of $20 \mathrm{~m}$ for indoor and $100 \mathrm{~m}$ for outdoor used. IEEE $802.11 \mathrm{~b} / \mathrm{g} / \mathrm{p}$ have been released in 1999, 2003 and 2009 respectively. The speed of these models are $11 \mathrm{Mbps}$, 54Mbps and 27Mbps. 802.11g model show the highest speed but 802.11 has the longest transmission rate which is $1000 \mathrm{~m}$ [16].

Due to the flexibility of drones' deployments and applications, few studies have been conducted to evaluate the communication performance in drones. However, the deployment of the drones in simulation studies were using different quantity of flying drones and mobility models. Thus, the optimum drone's flying pattern is hardly found.

\section{B. Mobility Model}

In [17] the authors present a static model with 9 drones flying in the area of one Square kilometer simulated in Java. This static model is implemented to provide a good network for mobile users when a disaster occurred as a backup network. The mobile users in the range of 50 to 100 people and the drone used an adhoc network with $300 \mathrm{~m}$ communication distance. The outcome of this pattern, they able to cover $98.59 \%$ of the considered area.

In [18] the authors deploy 5 drones with a Jaccard model flying pattern. They simulate a disaster scenario with 125 victims randomly placed around $1 \mathrm{~km}$ by $1 \mathrm{~km}$ area using python and built the flying model using BonnMotion tools. The communication range of the drone is $250 \mathrm{~m}$. They tested the flying model with 3 different Jaccard threshold which are 0.2 , 0.5 and 0.8 . The results show that the highest covered area is $80.93 \%$ gained using threshold 0.8 while threshold 0.5 and 0.2 are able to cover $60.67 \%$ and $42.95 \%$ area respectively.

Similar to previous work, the authors used BonnMotion tools to design an Enhanced Manhattan model with 10 to 100 drones using NS2.35 software [19]. The drones used 802.11 wireless protocol and deployed around $15.229 \mathrm{~km}$ by $1.3 \mathrm{~km}$ area to study Ad-hoc On Demand Vector (AODV), Destination Sequenced Distance Vector (DSSV) and Dynamic Source Routing (DSR) performance. However, the designed mobility model able to cover $56.33 \%$ of the considered area.

Another group of researchers examined the network performance for drones during land surveying [20] and disaster environment [21]. In [20], they deployed 5 to 30 drones within a $4 \mathrm{~km}$ by $4 \mathrm{~km}$ area in NS3 simulator with $\mathrm{C}++$ tools. The flying patterns used are random, force-based and alpha-based mobility models. IEEE 802.11 Wi-Fi model is attached to the drones with a $400 \mathrm{~m}$ wireless range. The simulation outcomes show that the alpha model able to cover $28.91 \%$ while the force model can cover $22.81 \%$ and the random model cover the least which is $1.27 \%$ area.

Meanwhile, as reported in [21], 16 drones were deployed in Matlab software and applied a similar concept as in [17] and known as matching game algorithm as well as for evaluation with minimum distance allocation method. The authors developed these algorithms to offer an instant communication network for a post-disaster environment. The drones used 802.11 as the communication medium. The simulation results show that it covered the considered area at $73.65 \%$ and $70.39 \%$ respectively.

However, 802.11b has been used in [22] to study the performance of mobile ad hoc tactile with two different types of network protocols and different mobility model with a maximum of 50 drones. The results showed that the random walk model covered $62.27 \%$ while the reference point group model covered $43.59 \%$ and the Manhattan Grid model covered the least which is $26.01 \%$ from $1 \mathrm{~km}$ by $1 \mathrm{~km}$ area.

In [23], 10 to 100 drones have been deployed in a $3.6 \mathrm{~km}$ by $1.2 \mathrm{~km}$ area with the random waypoint model to investigate the transmission range performance of $802.11 \mathrm{~g}$ using AODV and OLSR routing protocols in FANET. The outcome of the study found that the mobility model covered $28.36 \%$ area only.

A smooth random walk model has been applied to deploy 20 to 140 drones to investigate the $802.11 \mathrm{p}$ performance between $2 \mathrm{D}$ and $3 \mathrm{D}$ in a $2 \mathrm{~km} * 2 \mathrm{~km}$ area [24]. However, this model able to covered half of the required area which is $52.84 \%$.

The studies from previous works show that their work could cover almost the whole considered area and enable 100 of mobile users to access the network created by 9 hovering drones. Meanwhile, they used hundreds of drones for an area less than $20 \mathrm{~km}^{2}$ and some of the work shows that the drone flies near another drone where it is not maximumly used of the drone's capability.

\section{Topology}

Research in [17] deployed drones with uniform $252 \mathrm{~m}$ distance side by side. Similar to [21], where the drones fly 400m apart from each other and remain at the position during the simulation. Meanwhile, other studies deployed drones nonuniform distance even though they deployed a number of drones at once.

Studies in [20] and [21] uses multihop to transmit the data collected from the furthest drones to the base station. Due to the network range limitation, multihop is used to notify the user.

\section{Methodology}

In this section, the methodology used to analyse the relationship between camera specifications and drones' flying height to coverage are is presented. Two different drones and camera specifications were used in this work. There are DJI Phantom 3 Standard and DJI Matrice 600 Pro. Figure 1 depicts the process flow for the analysis. In contrast to other simulation works, this project utilized a single drone in the surveying process. Different flying patterns were tested on both specifications and different flying height. The objective of this part is to obtain the optimum technical specification in achieving the highest coverage area. Table 1 tabulates the drone's and camera's specifications. This study assumed that the camera is facing directly to the ground. Other details are as described in Table I and II. 


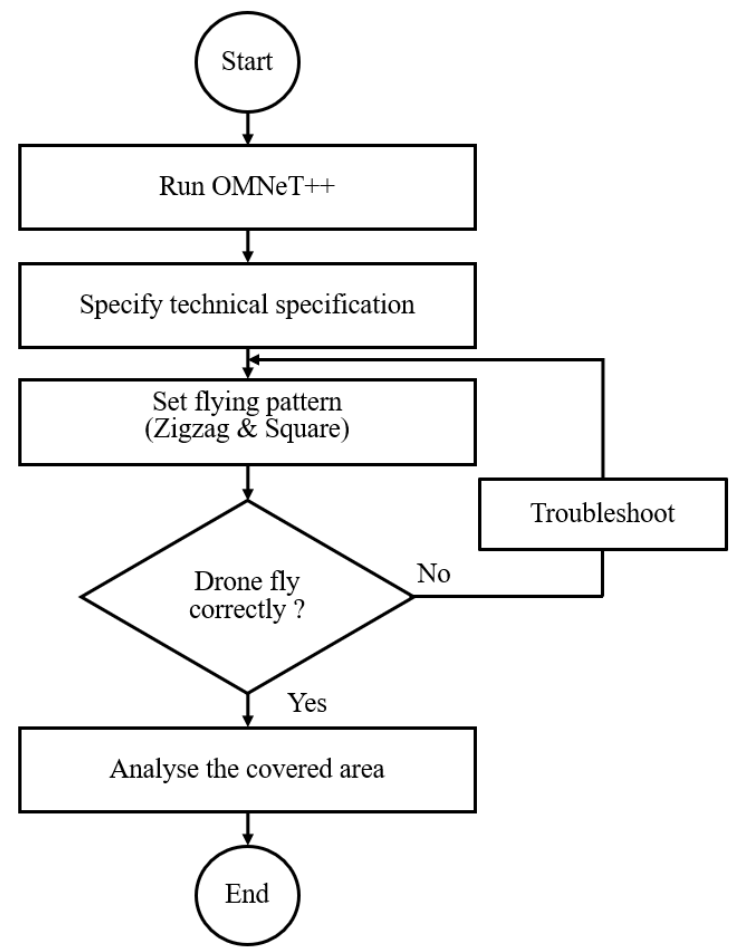

Figure 1: Flow of the project

Table I shows that two type of drones and cameras from the DJI commercial brand. DJI Phantom 3 Standard is a quadcopter model while DJI Matrice 600 Pro is a hexacopter model. The flight time for Phantom 3 is around 25 minutes while Matrice 600 Pro available to fly around 35 minutes with minimum payloads.

TABLE I

TyPes OF DRONES With FIELD OF VIEW AND FLIGHT TIME

\begin{tabular}{ccc}
\hline Specifications & DJI Phantom 3 & DJI Matrice 600 \\
& Standard & Pro \\
\hline Camera & N/A & Z30 \\
FOV & $94^{\circ}$ & $63.7^{\circ}$ \\
Flight Time & App. $25 \mathrm{~min}$ & $35 \mathrm{~min}$ \\
\hline
\end{tabular}

This project is simulated using $\mathrm{OMNeT}++$ version 5.2.1 with extension tools Inet version 3.6.3. Two flying patterns named Zigzag and Square is used to analyse the covered area for $10 \mathrm{~m}$ and $20 \mathrm{~m}$ height. A drone can fly legally at $120 \mathrm{~m}$ as the highest altitude and $10 \mathrm{~m}$ height is the minimum height to fly [25][26]. The decision of $10 \mathrm{~m}$ and $20 \mathrm{~m}$ height is based on the fieldwork test conducted by another research team member. Their study has found out that the best image captured was when the drone flies less than $30 \mathrm{~m}$.

TABLE II

\begin{tabular}{cc}
\multicolumn{2}{c}{ SIMULATION PARAMETERS } \\
\hline Parameters & Values \\
\hline Simulator & OMNeT++ \\
Simulation Area & $200 \mathrm{~m} \times 200 \mathrm{~m}$ \\
Simulation Time & $93 \mathrm{~s}($ Zigzag) \\
& $115 \mathrm{~s}$ (Square) \\
Distance Travel & $1290 \mathrm{~m}$ (Zigzag) \\
& $1600 \mathrm{~m}$ (Square) \\
Number of UAV & 1 \\
Speed of UAV & $14 \mathrm{~m} / \mathrm{s}$ \\
\hline
\end{tabular}

\section{A. Field Of View}

Figure 2 illustrates the condition of the drone above the ground and the view range from a camera attached to the drone. The width of the FOV influenced by the FOV angle of the camera itself and the distance between the lens and ground.

It is important to identify the FOV angle of the camera. Every camera has a different FOV angle and the detail is provided by the manufacture. Next, divide the FOV angle by 2 and further compute the height, $h$ for the drone to be evaluated during flying. Further, the FOV is calculated as shown in Equation (1), and from here the value of $y$ will be computed along with the total width of FOV upon multiplication with 2 .

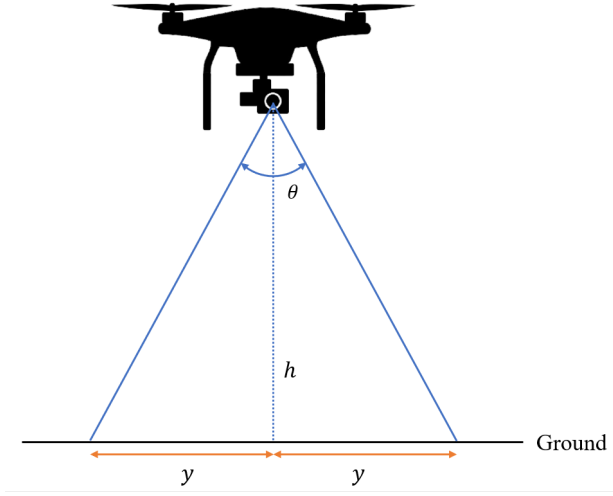

Figure 2: Illustration of the field of view (FOV) of drone's camera from above

$y=(\tan \theta)(h)$

To obtain the FOV as shown in Equation (1), we need to find the $y$ value in meter. Next, we multiply with 2 to get the total width of FOV.

\section{B. Percentage of Covered Area}

The percentage of the covered area is significant in the search and rescue mission. The flying pattern is unsatisfy to be implemented when it could not able to cover as much as it can to screening the disaster area. After the width of FOV has been identified, then we will mark along the flight path using the previous FOV width. Next, we will use Imagej software to convert the image to black and white then, extract the image to gain the highlighted area size.

\section{Results and Discussion}

This section presents the results followed by a brief discussion on the finding. In this study, flying pattern Zigzag and Square were used. This is based on the promising result from previous works on the flying pattern.

Figure 3 and 4 show a flying pattern named Zigzag based on numerical analysis and simulation using $\mathrm{OMNeT}++$. The total distance of the flight is $1290 \mathrm{~m}$ at $10 \mathrm{~m}$ above the ground. The black-colored drone is at the endpoint of the flying pattern and the black line before it, is the flight traces.

The yellow highlighted along the flight traces is the width of FOV which represented as the covered area of each camera. The width of FOV using camera Phantom 3 Standard is wider than using camera Z30. Figure 5 and 6 are Square flying pattern and 
fly at the same altitude as Figure 3 and 4. The result obtained also similar to the previous pattern in which the FOV width of Figure 3 and 5 is wider than Figure 4 and 6.

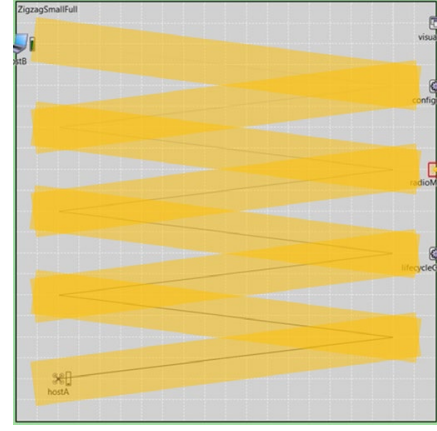

Figure 3:Zigzag pattern at $10 \mathrm{~m}$ using camera Phantom 3 Standard

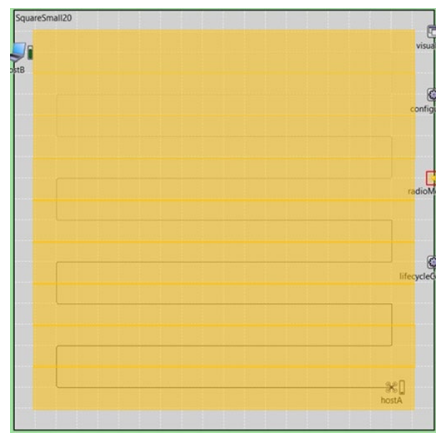

Figure 5: Square pattern at $10 \mathrm{~m}$ using camera Phantom 3 Standard

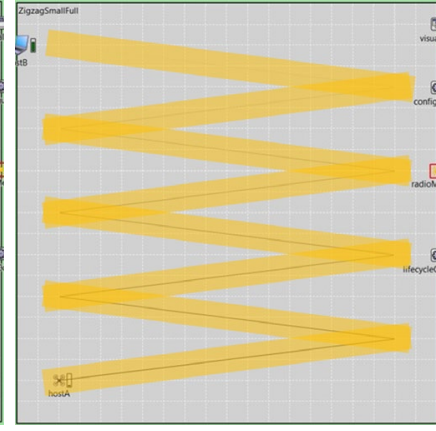

Figure 4: Zigzag pattern at $10 \mathrm{~m}$ using camera Z30

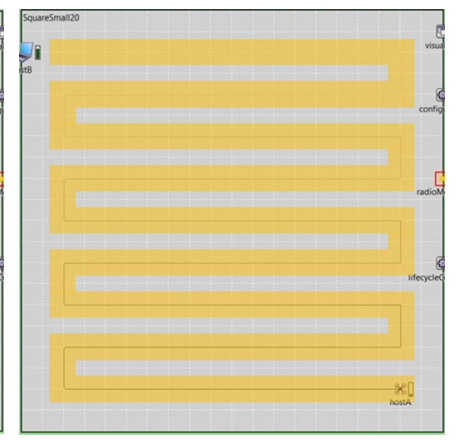

Figure 6:Square pattern at $10 \mathrm{~m}$ using camera Z30 for Square pattern during simulation. Moreover, at this altitude Square flying pattern able to cover the whole area with the same distance travel as the previous altitude.

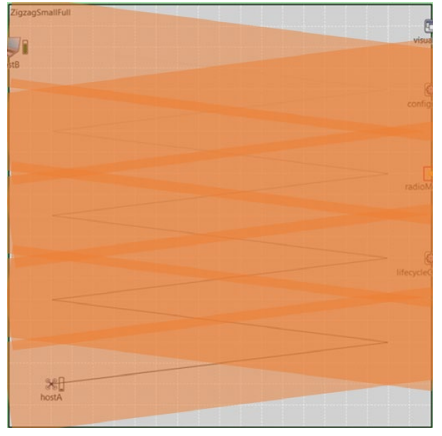

Figure 7:Zigzag pattern at $20 \mathrm{~m}$ using camera Phantom 3 Standard

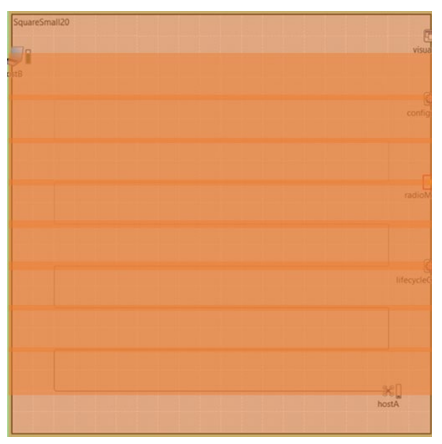

Figure 9: Square pattern at $20 \mathrm{~m}$ using camera Phantom 3 Standard

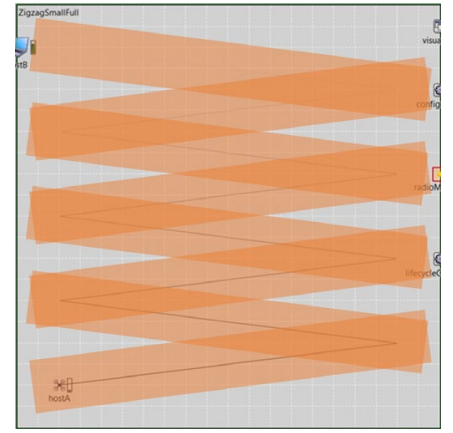

Figure 8: Zigzag pattern at 20m using camera $\mathrm{Z} 30$

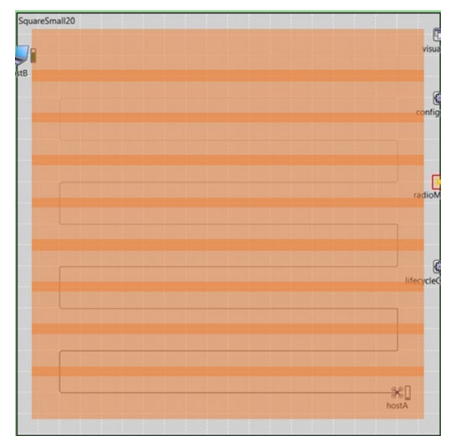

Figure 10: Square pattern at 20m using camera Z30
Referred to Figure 3 to 6, the Zigzag pattern travels a shorter distance than the Square pattern, which means it uses less energy and takes less time. However, the Square pattern is capable of covering the entire considered area.

In addition, Figures 7 and 8 show the Zigzag flying pattern at $20 \mathrm{~m}$ and the FOV width highlighted with red colour. However, the FOV of Phantom 3 Standard is greater as compared to Z30.

Similar to the Square pattern in Figure 9 and 10 flies at $20 \mathrm{~m}$. the width of FOV is wider compared to the FOV width from $10 \mathrm{~m}$ altitudes. This is because the area is captured from $20 \mathrm{~m}$ above the ground. The distance between the lens and ground is double as compared to Figures 9 and 10.

Referred to Figure 7 to 10, the FOV width is redundant in a certain area. Due to the same flight distance as for the $10 \mathrm{~m}$ altitude scenario, the width of FOV is double and overlapped with other paths thus we can reduce the distance when drone fly at higher altitude.

Figures 11 presents an analysis of the percentage of coverage area captured by $10 \mathrm{~m}$ altitude. The percentage of the covered area obtained from camera Phantom 3 Standard increased by $36.64 \%$ for Zigzag pattern and $39.45 \%$ for Square pattern during simulation.

Next, Figure 12 also presents an analysis of the percentage of coverage area captured by camera Phantom 3 Standard and Matrice 600 Pro but at a higher altitude which is $20 \mathrm{~m}$. The percentage of the covered area obtained from camera Phantom 3 Standard increased by $40.66 \%$ for Zigzag pattern and $39.84 \%$
Percentage of Covered Area of Each Patterns

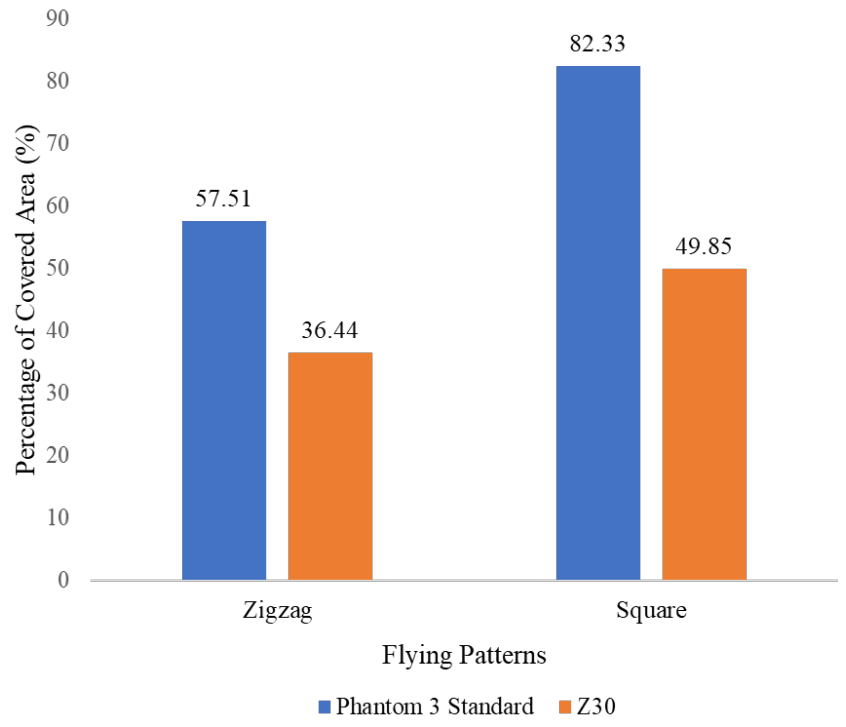

Figure 11: The percentage of coverage area from both drones and flying patterns at $10 \mathrm{~m}$ heights. 
Percentage of Covered Area of Each Patterns

120

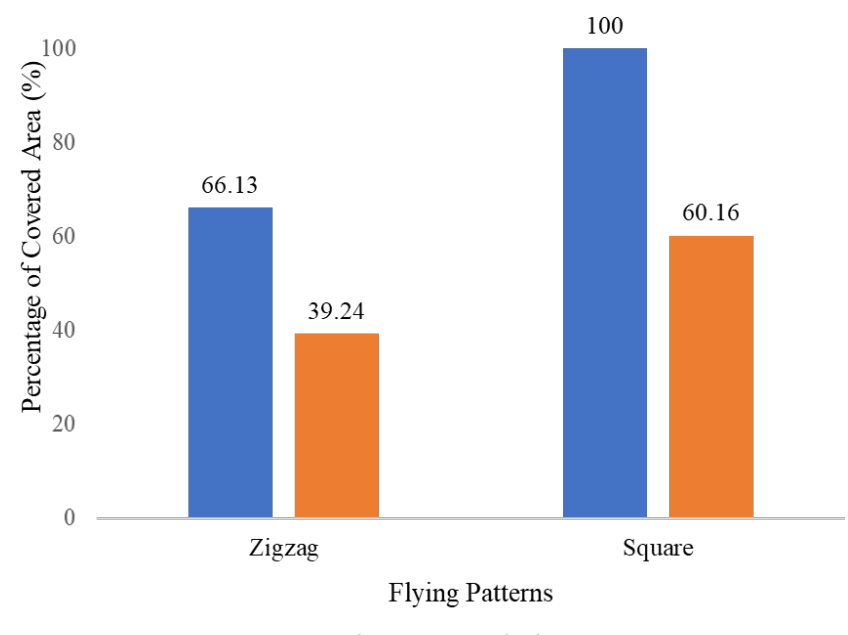

- Phantom 3 Standard $\square \mathrm{Z} 30$

Figure 12:The percentage of coverage area from both drones and flying patterns at $20 \mathrm{~m}$ heights

A camera attached to DJI Phantom 3 Standard shows the best result as compared to camera $\mathrm{Z} 30$ in all scenes. This is because the FOV angle of the Phantom 3 Standard camera itself is wider than Z30.

This project aims to identify the best flying pattern where it capable of screening the entire considered area, especially for disaster area to find the victims. However, few important criteria need to be focused such as the camera's specification and altitude for the flying drone. In view of a disaster area size in real life might be larger than $10 \mathrm{~km}^{2}$ thus flying pattern with the best camera specification at optimum altitude will assist the search and rescue mission efficiently. Due to the high simulation time for higher coverage area, the study will limit to a maximum of $1 \mathrm{~km}$ radius only.

\section{CONCLUSION}

In conclusion, two different technical camera specification and two drone flying patterns are analysed in this paper. This paper presents part of the overall study on the optimum flying pattern for a drone in a disaster area. The camera technical specification and drone flying height were given by another group of the research team. The DJI Phantom 3 Standard with its high-performance camera overperformed the DJI Matrice 600 Pro at $10 \mathrm{~m}$ and $20 \mathrm{~m}$ heights. The result shows that the height of the flying drone will affect the coverage area of the scene and the inclusion region will be more extensive with a more extensive angle of FOV. Future work will focus on reducing the redundancy in the coverage thus ensure optimum energy consumption.

\section{ACKNOWLEDGEMENT}

The authors would like to thank the Ministry of Education (MOE) Malaysia for providing the grant 600-IRMI/TRGS 5/3 (001/2019)-1, and the Research Management Centre (RMC) of
Universiti Teknologi MARA for supporting this research work.

\section{REFERENCES}

[1] M. C. Elish, "Remote Split: A History of US Drone Operations and the Distributed Labor of War," Sci. Technol. Hum. Values, vol. 42, no. 6, pp. 1100-1131, 2017, doi: 10.1177/0162243917731523.

[2] A. K. Puttock, A. M. Cunliffe, K. Anderson, and R. E. Brazier, "Aerial photography collected with a multirotor drone reveals impact of Eurasian beaver reintroduction on ecosystem structure," J. Unmanned Veh. Syst., vol. 3, no. 3, pp. 123-130, 2015, doi: 10.1139/juvs-2015-0005.

[3] E. Cheng, Aerial Photography and Videography Using Drones. Peachpit Press, 2016.

[4] U. Jain, M. Rogers, and E. T. Matson, "Drone forensic framework: Sensor and data identification and verification," in SAS 2017 - 2017 IEEE Sensors Applications Symposium, Proceedings, 2017, doi: 10.1109/SAS.2017.7894059.

[5] K. E. Joyce, S. Duce, S. M. Leahy, J. Leon, and S. W. Maier, "Principles and practice of acquiring drone-based image data in marine environments," Mar. Freshw. Res., vol. 70, no. 7, pp. 952-963, 2019, doi: 10.1071/MF17380.

[6] B. Potter, G. Valentino, L. Yates, T. Benzing, and A. Salman, "Environmental Monitoring Using a Drone-Enabled Wireless Sensor Network," 2019 Syst. Inf. Eng. Des. Symp., pp. 1-6, 2019.

[7] R. I. Da Silva and M. A. Nascimento, "On best drone tour plans for data collection in Wireless Sensor Network," Proc. ACM Symp. Appl. Comput., vol. 04-08-Apri, pp. 703-708, 2016, doi: $10.1145 / 2851613.2851854$.

[8] T. Moribe, H. Okada, K. Kobayashl, and M. Katayama, "Combination of a wireless sensor network and drone using infrared thermometers for smart agriculture," CCNC 2018 - 2018 15th IEEE Annu. Consum. Commun. Netw. Conf., vol. 2018-Janua, pp. 1-2, 2018, doi: 10.1109/CCNC.2018.8319300.

[9] C. Seiber, D. Nowlin, B. Landowski, and M. E. Tolentino, "Tracking hazardous aerial plumes using IoT-enabled drone swarms," IEEE World Forum Internet Things, WF-IoT 2018 - Proc., vol. 2018-Janua, pp. 377382, 2018, doi: 10.1109/WF-IoT.2018.8355118.

[10] S. Oleksyn, L. Tosetto, and V. Raoult, "Drone-Based Tracking of the Fine-Scale Movement of a Coastal Stingray ( Bathytoshia brevicaudata )," Remote Sens., vol. 13, no. 40, pp. 1-24, 2021.

[11] R. F. Araujo et al., "Integrating high resolution drone imagery and forest inventory to distinguish canopy and understory trees and quantify their contributions to forest structure and dynamics," PLOS ONE.

[12] H. Nawaz, H. M. Ali, and S. ur R. Massan, "A study of mobility models for UAV communication networks," no. 2, pp. 276-297, 2019, doi: http://dx.doi.org/10.17993/3ctecno.2019.specialissue2.276-297 1.

[13] O. S. Oubbati, M. Atiquzzaman, and S. Member, "Routing in Flying Ad hoc Networks : Survey, constraints and future challenge perspectives," IEEE Access, vol. PP, no. June, p. 1, 2019, doi: 10.1109/ACCESS.2019.2923840.

[14] M. Shahir, H. Salem, F. Hafizhelmi, and K. Zaman, "Effectiveness of Human Detection from Aerial Images Taken from Different Heights," doi: 10.18421/TEMxx-xx.

[15] A. Nedjati, G. Izbirak, B. Vizvari, and J. Arkat, "Complete coverage path planning for a multi-UAV response system in post-earthquake assessment," Robotics, vol. 5, no. 4, 2016, doi: 10.3390/robotics5040026.

[16] Y. Wang, X. Duan, D. Tian, G. Lu, and H. Yu, "Throughput and Delay Limits of 802.11p and its Influence on Highway Capacity," Procedia Soc. Behav. Sci., vol. 96, no. Cictp, pp. 2096-2104, 2013, doi: 10.1016/j.sbspro.2013.08.236.

[17] M. Tropea and P. Fazio, "A Simulator for Creating Drones Networks and Providing Users Connectivity," in 2019 IEEE/ACM 23rd International Symposium on Distributed Simulation and Real Time Applications (DSRT), 2019.

[18] J. Sanchez-Garcia, J. M. Garcia-Campos, S. L. Toral, D. G. Reina, and F. Barrero, "A Self Organising Aerial Ad Hoc Network Mobility Model for Disaster Scenarios," in 2015 International Conference on Developments of E-Systems Engineering (DeSE), Dec. 2015, pp. 35-40, doi: 10.1109/DeSE.2015.12.

[19] S. Kour and J. S. Ubhi, "Performance analysis of mobile nodes in mobile ad-hoc networks using enhanced manhattan mobility model," J. Sci. Ind. Res., vol. 78, pp. 69-72, 2019.

[20] M. Messous, S. Senouci, and H. Sedjelmaci, "Network Connectivity and 
Area Coverage for UAV Fleet Mobility Model with Energy Constraint," in IEEE Wireless Communications and Networking Conference (WCNC 2016) - Track 4 - Services, Applications, and Business, 2016.

[21] K. Ali, H. X. Nguyen, Q. Vien, P. Shah, and M. Raza, "Deployment of Drone-Based Small Cells for Public Safety Communication System," IEEE Syst. J., pp. 1-10, 2019.

[22] J. Arshad, M. Ajmal, K. Salah, and R. Iqbal, "Performance analysis of content discovery for ad-hoc tactile networks," Futur. Gener. Comput. Syst., vol. 94, pp. 726-739, 2019, doi: 10.1016/j.future.2018.11.037.

[23] A. V. Leonov, G. A. Litvinov, and D. A. Korneev, "Simulation and analysis of transmission range effect on AODV and OLSR routing protocols in Flying Ad Hoc Networks (FANETs) formed by mini-UAVs with different node density," in 2018Systems of Signal Synchronization, Generating and Processing in Telecommunications, SYNCHROINFO 2018, 2018, doi: 10.1109/SYNCHROINFO.2018.8457014.

[24] N. Lin, F. Gao, L. Zhao, A. Al-dubai, and Z. Tan, "A 3D smooth random walk mobility model for FANETs," in 2019 IEEE 21st International Conference on High Performance Computing and Communications; IEEE 17th International Conference on Smart City; IEEE 5th International Conference on Data Science and Systems (HPCC/SmartCity/DSS), 2019, pp. 460-467, doi: 10.1109/HPCC/SmartCity/DSS.2019.00075.

[25] A. G. Perera, Y. W. Law, and J. Chahl, "Drone-action: An outdoor recorded drone video dataset for action recognition," Drones, vol. 3, no. 4, pp. 1-16, 2019, doi: 10.3390/drones3040082.

[26] M. Ishigami and T. Sugiyama, "A Novel Drone's Height Control Algorithm for Throughput Optimization in Disaster Resilient Network," IEEE Trans. Veh. Technol., vol. 69, no. 12, pp. 16188-16190, 2020, doi: 10.1109/TVT.2020.3032151.

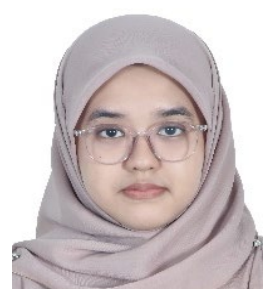

Intan Nabina Azmi was born in Johor, Malaysia, in 1995. She obtained her B. Eng. (Hons) Electronics Engineering, minor in computer from Universiti Teknologi MARA (UiTM), Shah Alam, Malaysia, in 2019. She obtained her Diploma in Electrical Engineering (Electronics) from Universiti Teknologi MARA (UiTM), Pasir Gudang, Malaysia, in 2016. She is pursuing a Master of Science in the Faculty of Electrical Engineering, Universiti Teknologi MARA (UiTM), Shah Alam, Malaysia. Her current research interests include information delivery for a wireless network, mobility model for drone and drone application at the disaster area.

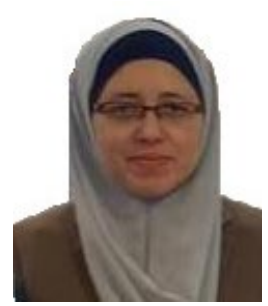

Yusnani Mohd Yussoff is a senior lecturer in the Faculty of Electrical Engineering, Universiti Teknologi MARA, Shah Alam Malaysia. She has 20 years working experience as a lecturer and researcher. Her research area focusses on Wireless Sensor Network, Trusted Authentication, Embedded Security and Internet of Things. She has graduated few $\mathrm{PhD}$ and Master students and currently involve few researchs related to the area. She is currently the head of Information, Security and Trusted Infrastructure Laboratory or InSTIL reseach group. She has authored and co-authored 41 indexed publications with SCOPUS h-index of 7. She is currently a member of IEEE, IAENG and Board of Engineer Malaysia.

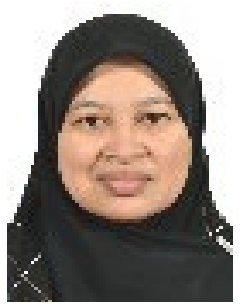

Murizah Kassim is currently working as Associate Professor at Faculty of Electrical Engineering, Universiti Teknologi MARA, Shah Alam, Selangor. She received her PhD in Electronic, Electrical and System Engineering in 2016 from the Faculty of Built Environment and Engineering, Universiti Kebangsaan Malaysia (UKM). She has published many indexed papers related to computer network, IoT, Web and Mobile development applications research. She experienced for 19 years in the technical team at the Centre for Integrated Information System, UiTM. She is also head of Enabling Internet of Things Technologies (ElIoTT) research group UiTM. She joined the academic since January 2009 and currently member of MBOT, IEEE, IET, IAENG and IACSIT organization.

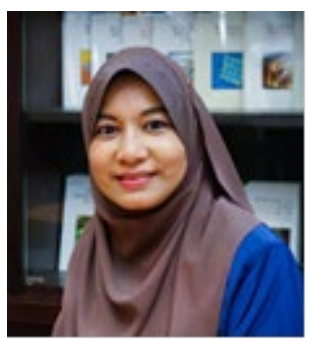

Nooritawati Md Tahir is a lecturer at the Faculty of Electrical Engineering, Universiti Teknologi MARA Selangor Malaysia. Her research interest is in the field of Image Processing, Pattern Recognition and Computational Intelligence. She has authored and coauthored more than 240 indexed publications with SCOPUS h-index of 16. In addition, she is a Certified Trainer for Postgraduate Training in Innovation and Entrepreneurship awarded by TCDUCD Dublin, Ireland, Stanford Faculty Fellow organised by Stanford Technology Ventures Program, Stanford University, US and Chartered Engineer (CEng) from the Institution of Engineering and Technology and Engineering Council UK as well as Senior Member (SMIEEE) of Institution of Electrical, Electronic Engineer (IEEE). 